\title{
P-Selectin: An Unpredicted Factor for Deep Vein Thrombosis after Total Hip Arthroplasty
}

\author{
Dongquan Shi, ${ }^{1,2}$ Xingquan $\mathrm{Xu},{ }^{1,2}$ Zhihong $\mathrm{Xu},{ }^{1}$ Takahiro Nakamura, ${ }^{3,4}$ Yong Pang, \\ Chen Yao, ${ }^{1}$ Feng Wang, ${ }^{1}$ Dongyang Chen, ${ }^{1}$ Jin Dai, ${ }^{1,2}$ and Qing Jiang ${ }^{1,2}$ \\ ${ }^{1}$ The Center of Diagnosis and Treatment for Joint Disease, Drum Tower Hospital Affiliated to Medical School of Nanjing University, \\ Nanjing, Jiangsu 210008, China \\ ${ }^{2}$ Laboratory for Bone and Joint Diseases, Australian-China Joint Centre, Model Animal Research Center, Nanjing University, Nanjing, \\ Jiangsu 210008, China \\ ${ }^{3}$ Laboratory for Mathematics, National Defense Medical College, 3-2 Namiki, Tokorozawa, Saitama 359-8513, Japan \\ ${ }^{4}$ Laboratory for Statistical Analysis, Center for Genomic Medicine, RIKEN, 4-6-1 Shirokanedai, Minato-ku, Tokyo 108-8639, Japan
}

Correspondence should be addressed to Qing Jiang; qingj@nju.edu.cn

Received 20 March 2014; Accepted 30 May 2014; Published 25 June 2014

Academic Editor: Kuo-Sheng Hung

Copyright (C) 2014 Dongquan Shi et al. This is an open access article distributed under the Creative Commons Attribution License, which permits unrestricted use, distribution, and reproduction in any medium, provided the original work is properly cited.

Introduction. Deep vein thrombosis (DVT) is a severe complication after total hip arthroplasty (THA). It leads to acute pulmonary embolism, a life-threatening disease. P-selectin is a $140-\mathrm{kDa}$ transmembrane glycoprotein. Elevated P-selectin was associated with 1.7 -fold increase in the risk of venous thrombosis. Materials and Methods. To confirm the association, a total of 91 subjects who received primary total hip arthroplasty using lateral approach performed by one skilled orthopedic surgeon were studied. All the patients were consecutively enrolled at the Center of Diagnosis and Treatment for Joint Diseases, Drum Tower Hospital affiliated to the Medical School of Nanjing University from 2010 to 2012. All the subjects received venography 3-5 days after operation. We measured P-selectin by means of a highly sensitive sandwich ELISA technique and a commercially available test reagent set. Results. No significant association was detected between P-selectin and DVT (all $P$ values $>0.05$ ). $\Delta$ sP-selectin was correlated with weight, APTT after operation, history of DVT, and diagnosis of primary disease ( $P$ values were $0.03,0.03,0.04$, and 0.02 , resp.). Conclusion. P-selectin may not be a predicted factor for deep vein thrombosis after total hip arthroplasty.

\section{Introduction}

Deep vein thrombosis (DVT) is a severe complication after total hip arthroplasty (THA). It leads to acute pulmonary embolism, a life-threatening disease. The prevalence of DVT after THA has ranged from 15 to $30 \%$ and 35 to $88 \%$ when prophylaxis has been used or not $[1,2]$. Pulmonary embolism, most commonly originating from deep venous thrombosis of the legs, ranges from asymptomatic, incidentally discovered emboli to massive embolism causing immediate death. Acute pulmonary embolism may occur rapidly and unpredictably and may be difficult to diagnose. Treatment can reduce the risk of death, and appropriate primary prophylaxis is usually effective. Early diagnosis has been reported to reduce VTE rate and mortality [3]. There are a lot of biomarkers identified for early diagnosis of or treatment for DVT [4].

Many studies have investigated the association of the cell adhesion molecule P-selectin with blood coagulation and thrombosis [5-10]. P-selectin is partially responsible for the activation of platelets and the adhesion of certain leukocytes and platelets to the endothelium [11]. Elevated P-selectin was associated with 1.7-fold increase in the risk of venous thrombosis [12]. The plasma concentration of Pselectin is largely related to platelet activation, and the levels of P-selectin are closely associated with the risk of venous thrombosis [11]. Interaction between P-selectin and its main counterreceptor on leukocytes, P-selectin glycoprotein ligand 1 (PSGL-1), leads to neutrophil and macrophage recruitment 
and, along with other mediators, induces leukocytes to generate procoagulant microparticles [13]. Furthermore, Pselectin triggers increased expression of tissue factor on monocytes [12] and mediates the transfer of tissue factor to platelets [14]. Tissue factor, the main initiator of coagulation in vivo, causes activation of the extrinsic pathway of the coagulation cascade. A recent study revealed that P-selectin induces phosphatidylserine exposure and increases surfacedependent thrombin generation on monocytes [15]. This property may represent an additional prothrombotic mechanism.

Possible roles for P-selectin in the pathogenesis of thrombosis were explored in several in vivo studies. Myers et al. $[16,17]$ demonstrated significantly lower thrombus weights in genetically modified animals that were deficient in Pand E-selectin compared with wild type control animals and showed that high circulating concentrations of P-selectin caused larger thrombi. Patients with venous thromboembolism (VTE) have demonstrated increased sP-selectin concentrations immediately after an acute event $[18,19]$ and at several months after VTE [20].

No study was reported on the association of P-selectin and acute DVT after THA. We conducted a case-control study to investigate the role of P-selectin in patients after operation of THA. Significant increase of solution P-selectin was detected after operation. However, no significant results were detected to confirm the previous reports on P-selectin as a predicate factor of DVT.

\section{Materials and Methods}

A total of 91 subjects who received primary total hip arthroplasty using lateral approach performed by one skilled orthopedic surgeon were studied. The prostheses are from the same company. All the patients were consecutively enrolled at the Center of Diagnosis and Treatment for Joint Diseases, Drum Tower Hospital affiliated to the Medical School of Nanjing University from 2010 to 2012. No subjects dropped out during the process of the study. The study was approved by the ethical committee of the participating institutions, and informed consent was obtained from all the patients.

2.1. Inclusion and Exclusion Criteria. The diagnoses of patients were fracture of femoral neck, avascular necrosis of femoral head, development of dysplasia of hip, osteoarthritis, and so forth. No revision hip arthroplasty was included.

All the patients received $0.3 \mathrm{~mL}$ of low-molecular-weight heparin subcutaneously once daily. Prophylaxis was continued until venography was performed.

Venography was routinely performed by one doctor and randomly reviewed by at least 2 vascular surgeons. DVT was diagnosed according to the Robinov group's criterion. If DVT was detected, conventional thrombolysis treatment was to be started. If not, patients would not receive any further anticoagulation treatment.

Age, sex, DVT related history, diabetes mellitus (DM), hypertension, cancer, hormone therapy, drug, and smoking history were recorded. We measured clinical and biochemical data (weight, height, ABO blood type, PT, APTT, INR, fibrinogen, RBC, PLT, D-dimer, triglyceride, and cholesterol) and surgery-related data (blood loss, duration of surgery, $\mathrm{EF}, \mathrm{Hb}$, drainage, and symptoms of DVT). The duration of surgery, blood loss, anesthesia, and drainage all were described.

We measured sP-selectin by means of a highly sensitive sandwich ELISA technique and a commercially available test reagent set (Human sP-Selectin/CD62P ELISA reagent set; R\&D Systems) according to the manufacturer's instructions. Duplicate measurements were carried out with $100 \mathrm{~mL}$ aliquots of plasma diluted 20 -fold into sample diluent included in the ELISA reagent set and the absorbance at $450 \mathrm{~nm}$ with a microplate reader was measured (MR7000; Dynex Technologies/Dynatech Laboratories). We read the sP-selectin concentration from a calibration curve generated with RevelationTM software (version G 3.2) from Dynex Technologies.

\section{Statistics}

We tested the association between the DVT and the Pselectin by using Mann-Whitney test. The differences in clinical factors between the DVT statuses were compared by Mann-Whitney and Fisher's exact tests. Mann-Whitney tests, Kruskal-Wallis tests, and tests of correlation coefficient were used for detecting association between the P-selectin and other clinical factors. For all calculations of statistical analysis, the software $\mathrm{R}$ was used.

\section{Results}

Finally, 91 patients (64 females and 27 males) with the age of $65 \pm 12.1$ years old joined the study. BMI of all the patients is $24.9 \pm 3.97 \mathrm{~kg} / \mathrm{m}^{2}$. The incidences of DM, HP, and history of DVT are $7.7 \%, 35.2 \%$, and $7.7 \%$, respectively. $71.4 \%(5 / 7)$ of the patients with history of DVT recurred. $8.8 \%(8 / 91)$ of the patients have the habit of smoking. $42.9 \%$ (39/91), $14.3 \%(13 / 91)$, and $27.5 \%(25 / 91)$ of the patients are diagnosed with osteoarthritis (OA), developmental dysplasia of the hip $(\mathrm{DDH})$, and fracture of the hip, respectively. $4 \pm 1$ days after THA, patients received low limb angiography routinely.

No difference was detected for all the demographic data in DVT and non-DVT groups (all $P$ values $>0.05$ ) except VAS score $(P=0.05)$ (Tables 1 and 2$)$.

The concentrations of all the patients pre-op and postop are $2.37 \pm 1.41$ and $2.53 \pm 1.49 \mathrm{ng} / \mathrm{mL}$, respectively. The concentrations of DVT group pre-op and post-op are $2.14 \pm$ 0.95 and $2.26 \pm 1.04 \mathrm{ng} / \mathrm{mL}$. And in non-DVT group preop and post-op, the concentrations are $2.43 \pm 1.50$ and $2.60 \pm 1.58 \mathrm{ng} / \mathrm{mL}$. When stratified by sex, in female, the concentrations are $2.39 \pm 1.41$ and $2.53 \pm 1.49 \mathrm{ng} / \mathrm{mL}$; in male, the concentrations of pre- and post-op are $2.32 \pm 1.01$ and $2.39 \pm 1.09$. No significant results were detected when we compare the difference between DVT and non-DVT groups 
TABLE 1: DVT versus the continuous covariates.

\begin{tabular}{|c|c|c|c|c|c|}
\hline \multirow{2}{*}{ Subjects } & \multicolumn{2}{|c|}{$\mathrm{DVT}=1$} & \multicolumn{2}{|c|}{$\mathrm{DVT}=2$} & \multirow{2}{*}{$P$ value } \\
\hline & Mean & S.D. & Mean & S.D. & \\
\hline Age & 65.12 & 12.47 & 66.50 & 10.57 & 0.85 \\
\hline Height & 161.10 & 8.21 & 160.76 & 8.51 & 0.87 \\
\hline Weight & 64.03 & 10.92 & 65.91 & 9.34 & 0.77 \\
\hline PT & 11.65 & 0.72 & 11.54 & 0.65 & 0.57 \\
\hline INR & 1.41 & 3.35 & 1.01 & 0.06 & 0.53 \\
\hline APTT & 25.54 & 5.43 & 26.27 & 3.48 & 0.59 \\
\hline $\mathrm{TT}$ & 17.99 & 2.24 & 18.22 & 2.17 & 0.59 \\
\hline $\mathrm{Fb}$ & 3.23 & 0.72 & 3.20 & 0.72 & 0.93 \\
\hline $\mathrm{Hb}$ & 127.11 & 15.77 & 128.50 & 14.51 & 0.65 \\
\hline PLT (pre) & 188.15 & 59.97 & 184.39 & 49.66 & 0.96 \\
\hline D-dimer & 0.29 & 0.55 & 0.20 & 0.29 & 0.33 \\
\hline $\mathrm{EF}$ & 59.33 & 10.89 & 60.25 & 2.67 & 0.25 \\
\hline ESR & 30.62 & 14.10 & 32.35 & 11.80 & 0.64 \\
\hline OP time & 117.64 & 33.61 & 108.82 & 30.03 & 0.39 \\
\hline Blood lost & 522.60 & 319.73 & 520.59 & 351.36 & 0.84 \\
\hline VAS & 3.54 & 1.16 & 4.12 & 1.22 & 0.05 \\
\hline RBC post-op & 3.53 & 0.50 & 3.57 & 0.51 & 0.89 \\
\hline $\mathrm{Hb}$ post-op & 106.35 & 13.50 & 104.15 & 27.98 & 0.67 \\
\hline PT post-op & 155.53 & 58.15 & 149.96 & 62.26 & 0.96 \\
\hline PT post-op & 12.11 & 0.84 & 11.92 & 0.71 & 0.46 \\
\hline INR post-op & 1.06 & 0.07 & 4.43 & 14.37 & 0.72 \\
\hline APTT post-op & 27.19 & 5.29 & 28.49 & 9.05 & 0.35 \\
\hline TT post-op & 15.71 & 1.75 & 21.31 & 22.96 & 0.34 \\
\hline Fb post-op & 4.12 & 0.68 & 9.34 & 21.14 & 0.09 \\
\hline
\end{tabular}

$P$ values were obtained by using Mann-Whitney test.

TABLE 2: DVT versus the discrete covariates.

\begin{tabular}{lc}
\hline Subjects & $P$ value \\
\hline Sex & 0.39 \\
DM & 0.35 \\
HP & 0.79 \\
Cardio & 0.58 \\
History of DVT & 0.35 \\
History of surgery & 0.30 \\
Smoke & 1.00 \\
Diagnosis & 0.43 \\
Blood type & 0.43 \\
Edema & 0.36 \\
\hline Pvalues wer obtain
\end{tabular}

$P$ values were obtained by using Fisher's exact test.

for pre- and postsurgery and amount of change of P-selectins (all $P$ values are $0.67,0.45$, and 0.98 ; Table 3 ).

4.1. sP-Selectin and Clinical Data. No clinical data (age, height, weight, PT, INR, APTT, etc.) was significantly correlated with sP-selectin before and after operation (Table 4).
TABLE 3: DVT versus p-selectin.

\begin{tabular}{lccccc}
\hline \multirow{2}{*}{ Subjects } & \multicolumn{2}{c}{ DVT $=1$} & \multicolumn{2}{c}{ DVT $=2$} & \multirow{2}{*}{ M value } \\
& Mean & S.D. & Mean & S.D. & \\
\hline P-selectin (pre) & 2.43 & 1.50 & 2.14 & 0.95 & 0.67 \\
$\begin{array}{l}\text { P-selectin (post) } \\
\begin{array}{l}\text { Amount of change of } \\
\text { p-selectins }\end{array}\end{array}$ & -0.17 & 0.57 & -0.12 & 0.33 & 0.98 \\
\hline
\end{tabular}

$P$ values were obtained by using Mann-Whitney test.

Weight, APTT after operation, history of DVT, and diagnosis of primary disease were significantly correlated with $\Delta \mathrm{sP}$ selectin ( $P$ values were $0.03,0.03,0.04$, and 0.02 , resp.). Correlation coefficients for weight, APTT after operation, and sP-selectin were 0.23 and -0.24 , respectively (Tables 5 , 6 , and 7).

\section{Discussion}

Diagnostic strategy using P-selectin testing for DVT is still controversial. Some groups reported that the P-selectin testing to the diagnostic algorithm has the potential to make the diagnosis of DVT more convenient and economical $[18,19,21]$. However, this is the first study that reported the relationship between P-selectin and DVT after THA. This study shows that no significant results were detected to confirm the previous reports on P-selectin as a predicate factor of DVT. DVT after THA is a kind of acute condition. It is different from aetiology of DVT with abnormalities of anticoagulant and procoagulant systems. sP-selectin, as a membrane component of the platelet alpha granule and endothelial cell Weibel-Palade body, may be involved in the acute DVT but did not play a key role in its pathology.

Elevated P-selectin was detected in postoperative group. However, we did not find increased P-selectin in DVT group compared to non-DVT group. Rectenwald et al. found Pselectin levels to be significantly elevated in patients with acute DVT confirmed by duplex ultrasound [18]. Angiography is the golden standard for DVT. In our daily work, duplex ultrasound is sensitive to central thrombosis but hardly detects peripheral thrombosis. That may be the reason why we did not get similar results.

No significant difference was detected for the demographic data between DVT and non-DVT groups. Only VAS score in DVT group was significantly higher than nonDVT group. Patient who felt more pain would not like to do more rehabilitation. Then, it caused higher prevalence of DVT. Age, sex, history of DVT, blood type, BMI, PT, INR, APTT, TT, Fb, Hb, D-dimer, EF, duration of surgery, and blood loss are not risk factors in our study. There is no significant correlation between demographic data and Pselectin levels for pre- and postoperations. $\Delta$ sP-selectin had a slightly positive correlation with weight and weak negative correlation with APTT level after operation. As platelet is activated in the pathogenesis of obesity and vascular disease, 
TABLE 4: P-selectin versus the continuous covariates.

\begin{tabular}{|c|c|c|c|c|c|c|}
\hline \multirow{2}{*}{ Subjects } & \multicolumn{2}{|c|}{ P-selectin (pre) } & \multicolumn{2}{|c|}{ P-selectin (post) } & \multicolumn{2}{|c|}{ Amount of change of P-selectins } \\
\hline & Correlation coefficients & $P$ value & Correlation coefficients & $P$ value & Correlation coefficients & $P$ value \\
\hline Age & 0.15 & 0.17 & 0.18 & 0.08 & -0.13 & 0.23 \\
\hline Height & -0.10 & 0.37 & -0.10 & 0.35 & 0.03 & 0.81 \\
\hline Weight & 0.01 & 0.93 & -0.07 & 0.50 & 0.23 & 0.03 \\
\hline $\mathrm{PT}$ & -0.13 & 0.23 & -0.14 & 0.17 & 0.07 & 0.53 \\
\hline INR & 0.06 & 0.59 & 0.10 & 0.37 & -0.12 & 0.25 \\
\hline APTT & -0.01 & 0.89 & 0.05 & 0.66 & -0.17 & 0.11 \\
\hline TT & 0.05 & 0.65 & 0.03 & 0.75 & 0.03 & 0.75 \\
\hline $\mathrm{Fb}$ & 0.06 & 0.56 & 0.02 & 0.88 & 0.12 & 0.25 \\
\hline $\mathrm{Hb}$ & -0.12 & 0.26 & -0.13 & 0.21 & 0.06 & 0.58 \\
\hline PLT (pre) & 0.13 & 0.21 & 0.08 & 0.43 & 0.12 & 0.26 \\
\hline D-dimer & -0.12 & 0.28 & -0.04 & 0.70 & -0.20 & 0.07 \\
\hline $\mathrm{EF}$ & -0.01 & 0.94 & -0.04 & 0.70 & 0.10 & 0.36 \\
\hline ESR & 0.03 & 0.81 & 0.03 & 0.79 & -0.02 & 0.88 \\
\hline OP time & 0.10 & 0.34 & 0.08 & 0.45 & 0.05 & 0.66 \\
\hline Blood lost & 0.13 & 0.22 & 0.10 & 0.36 & 0.08 & 0.48 \\
\hline VAS & -0.12 & 0.25 & -0.07 & 0.51 & -0.13 & 0.23 \\
\hline RBC post-op & -0.09 & 0.43 & -0.05 & 0.63 & -0.07 & 0.53 \\
\hline Hb post-op & -0.03 & 0.77 & 0.04 & 0.72 & -0.17 & 0.12 \\
\hline PT post-op & 0.06 & 0.60 & 0.05 & 0.68 & 0.02 & 0.84 \\
\hline PT post-op & -0.15 & 0.15 & -0.20 & 0.06 & 0.15 & 0.16 \\
\hline INR post-op & 0.03 & 0.78 & 0.00 & 1.00 & 0.08 & 0.45 \\
\hline APTT post-op & 0.02 & 0.83 & 0.10 & 0.33 & -0.24 & 0.03 \\
\hline TT post-op & 0.06 & 0.59 & 0.01 & 0.89 & 0.11 & 0.29 \\
\hline Fb post-op & 0.02 & 0.83 & 0.00 & 0.98 & 0.07 & 0.51 \\
\hline
\end{tabular}

TABLE 5: P-selectin (pre) versus the other discrete covariates.

\begin{tabular}{|c|c|c|c|c|c|c|c|c|c|c|c|c|}
\hline \multirow{3}{*}{ Subjects } & \multicolumn{10}{|c|}{ Categories } & \multirow{3}{*}{$P$ value } & \multirow{3}{*}{ Categorization } \\
\hline & \multicolumn{2}{|c|}{0} & \multicolumn{2}{|c|}{1} & \multicolumn{2}{|c|}{2} & \multicolumn{2}{|c|}{3} & \multicolumn{2}{|c|}{4} & & \\
\hline & Mean & S.D. & Mean & S.D. & Mean & S.D. & Mean & S.D. & Mean & S.D. & & \\
\hline DVT & 2.43 & 1.50 & 2.14 & 0.95 & & & & & & & 0.67 & Normal $=0, \mathrm{DVT}=1$ \\
\hline Sex & 2.39 & 1.55 & 2.32 & 1.01 & & & & & & & 0.72 & Female $=0$, male $=1$ \\
\hline $\mathrm{DM}$ & 2.41 & 1.44 & 1.95 & 0.94 & & & & & & & 0.40 & \\
\hline $\mathrm{HP}$ & 2.31 & 1.09 & 2.49 & 1.87 & & & & & & & 0.58 & \\
\hline Cardio & 2.41 & 1.43 & 1.65 & 0.65 & & & & & & & 0.13 & \\
\hline History of DVT & 2.33 & 1.34 & 2.77 & 2.08 & & & & & & & 0.78 & \\
\hline History of surgery & 2.20 & 1.14 & 2.57 & 1.69 & & & & & & & 0.18 & \\
\hline Smoke & 2.39 & 1.44 & 2.17 & 1.02 & & & & & & & 0.82 & \\
\hline Diagnosis & 2.57 & 1.77 & 1.77 & 0.93 & 2.32 & 1.02 & 2.81 & 1.67 & 2.29 & 0.80 & 0.33 & \\
\hline Blood type & 2.15 & 0.87 & 2.31 & 1.16 & 2.95 & 2.53 & 2.43 & 1.62 & & & 0.89 & $\mathrm{~A}=0, \mathrm{~B}=1, \mathrm{AB}=2, \mathrm{O}=3$ \\
\hline Edema & 2.26 & 1.21 & 2.55 & 1.69 & 2.37 & 1.60 & 1.03 & & & & 0.39 & Light $=1$, middle $=2$, severe $=3$ \\
\hline
\end{tabular}

it is possible to have higher P-selectin level in DVT patients. smoke is a critical responsibility for DVT in the previous reports [21]. However, we did not find it in the present study, either for sex.

\section{Conclusion}

P-selectin may not be a predicted factor for deep vein thrombosis after total hip arthroplasty. 
TABLE 6: P-selectin (post) versus the other discrete covariates.

\begin{tabular}{|c|c|c|c|c|c|c|c|c|c|c|c|c|}
\hline \multirow{3}{*}{ Subjects } & \multicolumn{10}{|c|}{ Categories } & \multirow{3}{*}{$P$ value } & \multirow{3}{*}{ Categorization } \\
\hline & \multicolumn{2}{|c|}{0} & \multicolumn{2}{|c|}{1} & \multicolumn{2}{|c|}{2} & \multicolumn{2}{|c|}{3} & \multicolumn{2}{|c|}{4} & & \\
\hline & Mean & S.D. & Mean & S.D. & Mean & S.D. & Mean & S.D. & Mean & S.D. & & \\
\hline DVT & 2.60 & 1.58 & 2.26 & 1.04 & & & & & & & 0.45 & Normal $=0, \mathrm{DVT}=1$ \\
\hline Sex & 2.59 & 1.64 & 2.39 & 1.09 & & & & & & & 0.89 & Female $=0$, male $=1$ \\
\hline $\mathrm{DM}$ & 2.59 & 1.51 & 1.91 & 1.26 & & & & & & & 0.19 & \\
\hline $\mathrm{HP}$ & 2.50 & 1.19 & 2.59 & 1.96 & & & & & & & 0.52 & \\
\hline Cardio & 2.58 & 1.51 & 1.65 & 0.89 & & & & & & & 0.11 & \\
\hline Histor of DVT & 2.53 & 1.47 & 2.58 & 1.85 & & & & & & & 0.67 & \\
\hline History of surgery & 2.35 & 1.30 & 2.72 & 1.71 & & & & & & & 0.35 & \\
\hline Smoke & 2.56 & 1.53 & 2.23 & 1.07 & & & & & & & 0.67 & \\
\hline Diagnosis & 2.58 & 1.79 & 1.77 & 1.03 & 2.78 & 1.18 & 3.00 & 1.77 & 2.47 & 1.17 & 0.12 & $\begin{array}{l}\mathrm{OA}=0, \mathrm{AVN}=1, \\
\text { fracture }=2, \mathrm{DDH}=3, \\
\text { others }=4\end{array}$ \\
\hline Blood type & 2.25 & 1.03 & 2.51 & 1.32 & 2.89 & 2.09 & 2.68 & 1.83 & & & 0.81 & $\begin{array}{l}A=0, B=1, A B=2, \\
O=3\end{array}$ \\
\hline Edema & 2.45 & 1.29 & 2.68 & 1.84 & 2.49 & 1.40 & 0.78 & & & & 0.50 & $\begin{array}{l}\text { Light }=1 \text {, middle }=2 \text {, } \\
\text { severe }=3\end{array}$ \\
\hline
\end{tabular}

Mann-Whitney test and Kruskal-Wallis test were used for obtaining $P$ values.

TABLE 7: Amount of change of P-selectins versus the other discrete covariates.

\begin{tabular}{|c|c|c|c|c|c|c|c|c|c|c|c|c|}
\hline \multirow{3}{*}{ Subjects } & \multicolumn{10}{|c|}{ Categories } & \multirow{3}{*}{$P$ value } & \multirow{3}{*}{ Categorization } \\
\hline & \multicolumn{2}{|c|}{0} & \multicolumn{2}{|c|}{1} & \multicolumn{2}{|c|}{2} & \multicolumn{2}{|c|}{3} & \multicolumn{2}{|c|}{4} & & \\
\hline & Mean & S.D. & Mean & S.D. & Mean & S.D. & Mean & S.D. & Mean & S.D. & & \\
\hline DVT & -0.17 & 0.57 & -0.12 & 0.33 & & & & & & & 0.98 & Normal $=0, \mathrm{DVT}=1$ \\
\hline Sex & -0.20 & 0.57 & -0.07 & 0.42 & & & & & & & 0.39 & Female $=0$, male $=1$ \\
\hline $\mathrm{DM}$ & -0.18 & 0.54 & 0.04 & 0.39 & & & & & & & 0.12 & \\
\hline HP & -0.19 & 0.56 & -0.10 & 0.46 & & & & & & & 0.53 & \\
\hline Cardio & -0.17 & 0.54 & 0.00 & 0.28 & & & & & & & 0.44 & \\
\hline History of DVT & -0.19 & 0.52 & 0.20 & 0.47 & & & & & & & 0.04 & \\
\hline History of surgery & -0.15 & 0.57 & -0.15 & 0.48 & & & & & & & 0.29 & \\
\hline Smoke & -0.17 & 0.52 & -0.06 & 0.59 & & & & & & & 0.58 & \\
\hline Diagnosis & 0.00 & 0.44 & -0.01 & 0.32 & -0.46 & 0.66 & -0.19 & 0.20 & -0.18 & 0.55 & 0.02 & $\begin{array}{l}\mathrm{OA}=0, \mathrm{AVN}=1, \\
\text { fracture }=2, \mathrm{DDH}=3, \\
\text { others }=4\end{array}$ \\
\hline Blood type & -0.10 & 0.51 & -0.20 & 0.45 & 0.06 & 0.67 & -0.25 & 0.55 & & & 0.65 & $\begin{array}{l}\mathrm{A}=0, \mathrm{~B}=1, \mathrm{AB}=2 \\
\mathrm{O}=3\end{array}$ \\
\hline Edema & -0.18 & 0.58 & -0.13 & 0.41 & -0.13 & 0.67 & 0.25 & & & & 0.62 & $\begin{array}{l}\text { Light }=1, \text { middle }=2 \\
\text { severe }=3\end{array}$ \\
\hline
\end{tabular}

Mann-Whitney test and Kruskal-Wallis test were used for obtaining $P$ values.

\section{Conflict of Interests}

The authors declare that they have no conflict of interests.

\section{Authors' Contribution}

Dongquan Shi and Qing Jiang conceived and designed the experiment. Yong Pang, Chen Yao, and Feng Wang performed the venography. Xingquan $\mathrm{Xu}$, Zhihong $\mathrm{Xu}$, and Jin Dai measured the level of P-selectin. Dongquan Shi and Takahiro Nakamura performed statistical analysis. Dongyang
Chen performed the total hip arthroplasty operation. Dongquan Shi wrote the paper. Qing Jiang supervised the whole study. All authors contributed to the final paper.

\section{References}

[1] M. T. Clarke, J. S. Green, W. M. Harper, and P. J. Gregg, "Screening for deep-venous thrombosis after hip and knee replacement without prophylaxis," Journal of Bone and Joint Surgery. British Volume, vol. 79, no. 5, pp. 787-791, 1997. 
[2] G. P. Clagett, F. A. Anderson, J. Heit, M. N. Levine, and H. B. Wheeler, "Prevention of venous thromboembolism," Chest Journal, vol. 108, supplement 4, pp. 312S-334S, 1995.

[3] P. S. Wells, D. R. Anderson, M. Rodger et al., "Evaluation of Ddimer in the diagnosis of suspected deep-vein thrombosis," The New England Journal of Medicine, vol. 349, no. 13, pp. 1227-1235, 2005.

[4] H. Hou, Z. Ge, P. Ying et al., "Biomarkers of deep venous thrombosis," Journal of Thrombosis and Thrombolysis, vol. 34, no. 3, pp. 335-346, 2012.

[5] K. Ley, "The role of selectins in inflammation and disease," Trends in Molecular Medicine, vol. 9, no. 6, pp. 263-268, 2003.

[6] R. P. McEver, "Adhesive interactions of leukocytes, platelets, and the vessel wall during hemostasis and inflammation," Thrombosis and Haemostasis, vol. 86, no. 3, pp. 746-756, 2001.

[7] J.-G. Geng, M. P. Bevilacqua, K. L. Moore et al., "Rapid neutrophil adhesion to activated endothelium mediated by GMP-140," Nature, vol. 343, no. 6260, pp. 757-760, 1990.

[8] B. Furie and B. C. Furie, "Role of platelet P-selectin and microparticle PSGL-1 in thrombus formation," Trends in Molecular Medicine, vol. 10, no. 4, pp. 171-178, 2004.

[9] J. Théorêt, D. Yacoub, A. Hachem, M. Gillis, and Y. Merhi, "P-selectin ligation induces platelet activation and enhances microaggregate and thrombus formation," Thrombosis Research, vol. 128, no. 3, pp. 243-250, 2011.

[10] K. Miszti-Blasius, I. B. Debreceni, S. Felszeghy, B. Dezso, and J. Kappelmayer, "Lack of P-selectin glycoprotein ligand-1 protects mice from thrombosis after collagen/epinephrine challenge," Thrombosis Research, vol. 127, no. 3, pp. 228-234, 2011.

[11] R. Fijnheer, C. J. M. Frijns, J. Korteweg et al., "The origin of P-selectin as a circulating plasma protein," Thrombosis and Haemostasis, vol. 77, no. 6, pp. 1081-1085, 1997.

[12] A. Celi, G. Pellegrini, R. Lorenzet et al., "P-selectin induces the expression of tissue factor on monocytes," Proceedings of the National Academy of Sciences of the United States of America, vol. 91, no. 19, pp. 8767-8771, 1994.

[13] P. André, D. Hartwell, I. Hrachovinová, S. Saffaripour, and D. D. Wagner, "Pro-coagulant state resulting from high levels of soluble P-selectin in blood," Proceedings of the National Academy of Sciences of the United States of America, vol. 97, no. 25, pp. 13835-13840, 2000.

[14] U. Rauch, D. Bonderman, B. Bohrmann et al., "Transfer of tissue factor from leukocytes to platelets is mediated by CD15 and tissue factor," Blood, vol. 96, no. 1, pp. 170-175, 2000.

[15] I. del Conde, F. Nabi, R. Tonda, P. Thiagarajan, J. A. López, and N. S. Kleiman, "Effect of P-selectin on phosphatidylserine exposure and surface-dependent thrombin generation on monocytes," Arteriosclerosis, Thrombosis, and Vascular Biology, vol. 25, no. 5, pp. 1065-1070, 2005.

[16] D. Myers Jr., D. Farris, A. Hawley et al., "Selectins influence thrombosis in a mouse model of experimental deep venous thrombosis," Journal of Surgical Research, vol. 108, no. 2, pp. 212221, 2002.

[17] D. D. Myers, A. E. Hawley, D. M. Farris et al., "P-selectin and leukocyte microparticles are associated with venous thrombogenesis," Journal of Vascular Surgery, vol. 38, no. 5, pp. 10751089, 2003.

[18] J. E. Rectenwald, D. D. Myers Jr., A. E. Hawley et al., "D-dimer, P-selectin, and microparticles: novel markers to predict deep venous thrombosis. A pilot study," Thrombosis and Haemostasis, vol. 94, no. 6, pp. 1312-1317, 2005.
[19] A. Smith, J. W. Quarmby, M. Collins, S. M. Lockhart, and K. G. Burnand, "Changes in the levels of soluble adhesion molecules and coagulation factors in patients with deep vein thrombosis," Thrombosis and Haemostasis, vol. 82, no. 6, pp. 1593-1599, 1999.

[20] A. D. Blann, W. M. P. Noteboom, and F. R. Rosendaal, "Increased soluble P-selectin levels following deep venous thrombosis: cause or effect?" British Journal of Haematology, vol. 108, no. 1, pp. 191-193, 2000.

[21] W. Ageno, P. Prandoni, E. Romualdi et al., "The metabolic syndrome and the risk of venous thrombosis: a case-control study," Journal of Thrombosis and Haemostasis, vol. 4, no. 9, pp. 1914-1918, 2006. 


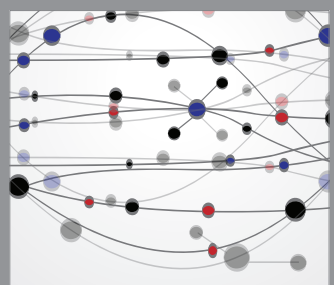

The Scientific World Journal
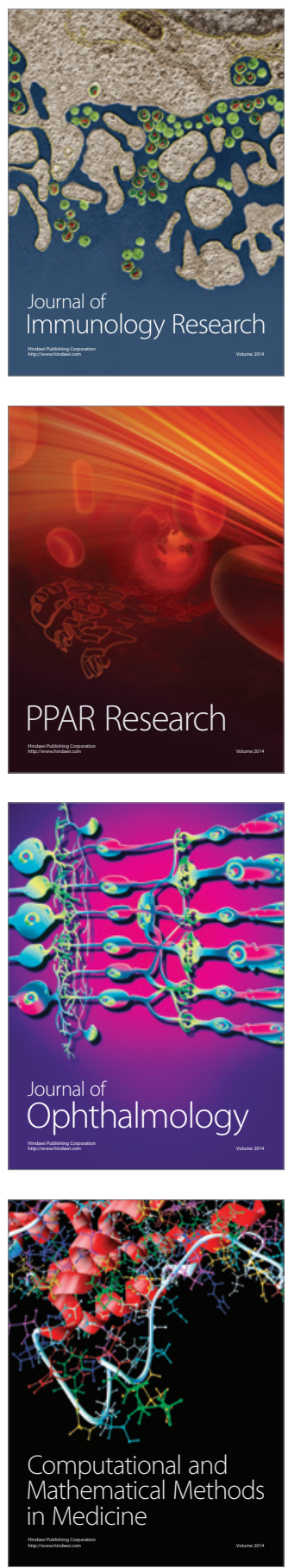

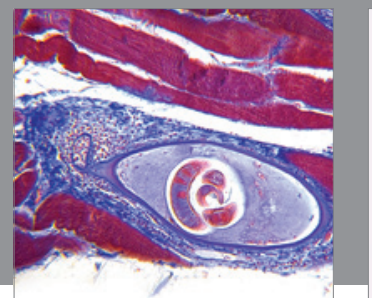

Gastroenterology

Research and Practice
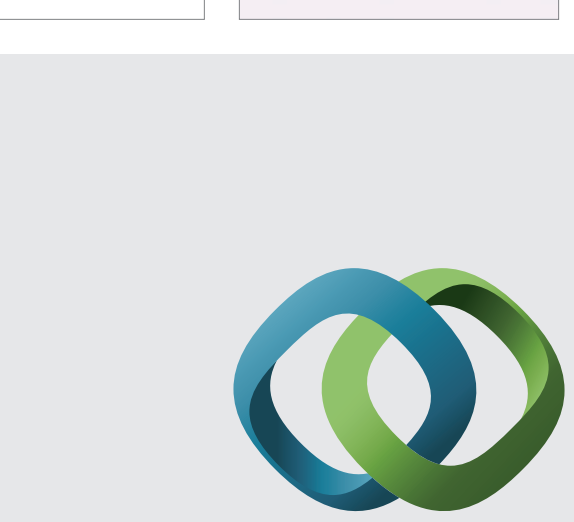

\section{Hindawi}

Submit your manuscripts at

http://www.hindawi.com
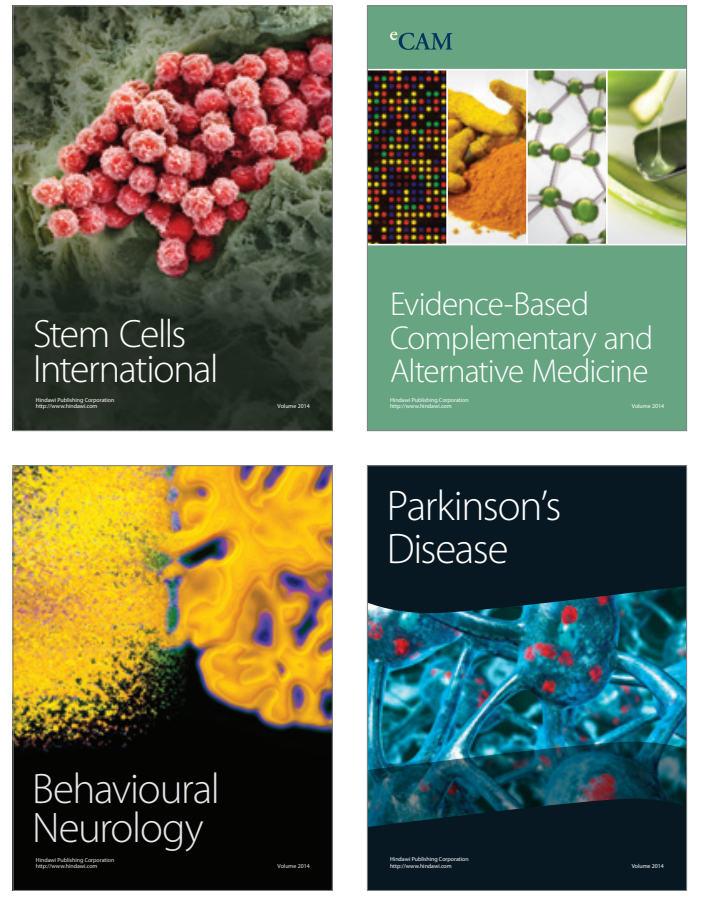
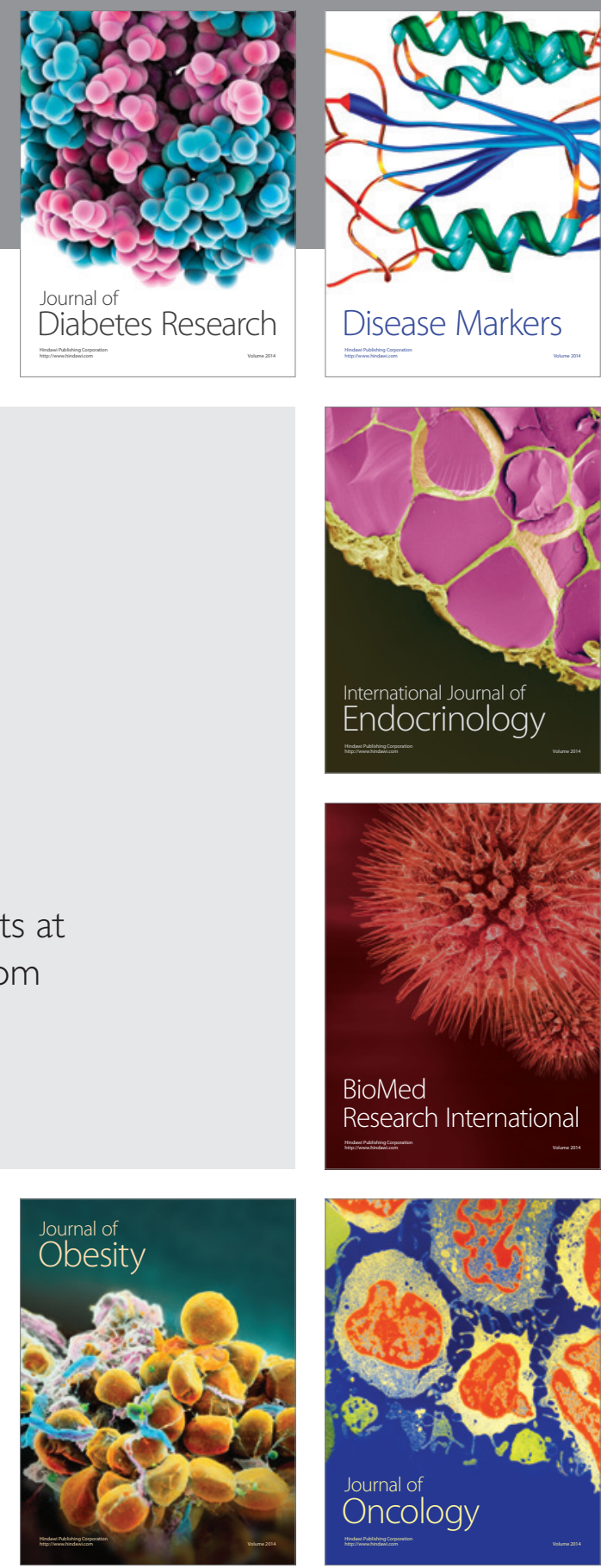

Disease Markers
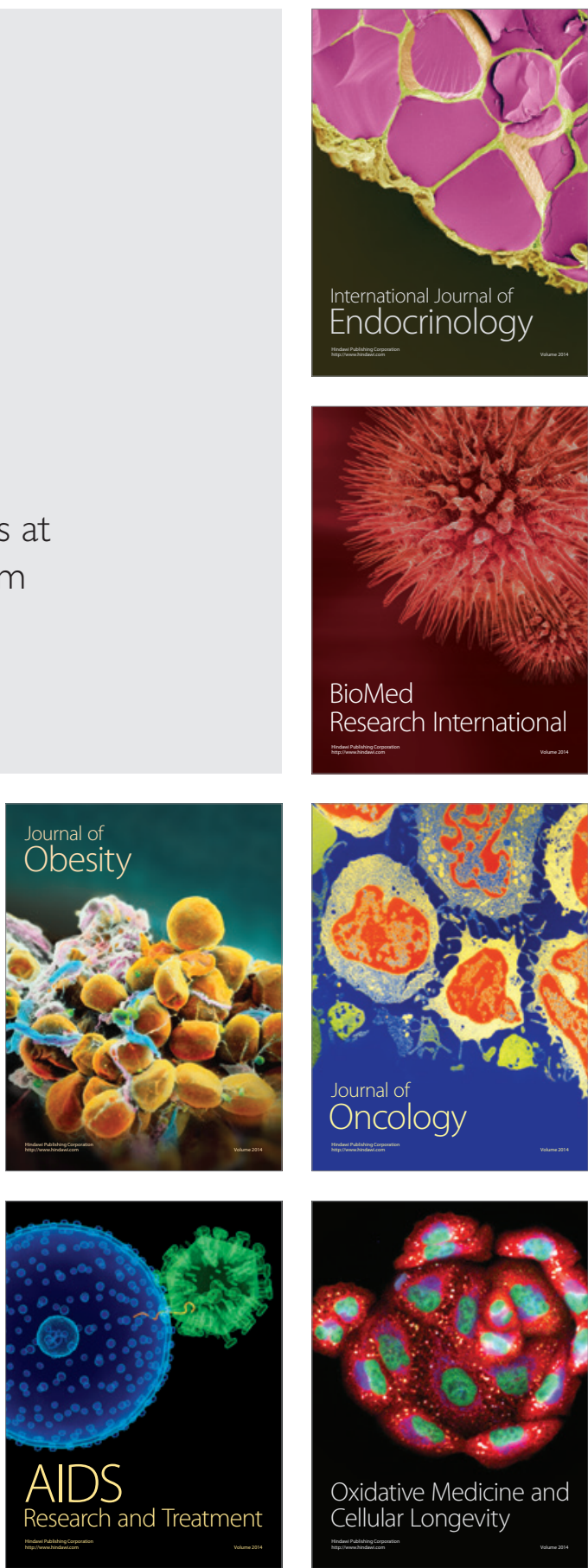